\title{
Zeta Potential of Extracellular Vesicles: Toward Understanding the Attributes that Determine Colloidal Stability
}

Getnet Midekessa, Kasun Godakumara, James Ord, Janeli Viil, Freddy Lättekivi, Keerthie Dissanayake, Sergei Kopanchuk, Ago Rinken, Aneta Andronowska, Sourav Bhattacharjee, Toonika Rinken, and Alireza Fazeli*

Cite This: ACS Omega 2020, 5, 16701-16710

Read Online

ACCESS | Lلll Metrics \& More | 回 Article Recommendations | (1) Supporting Information

ABSTRACT: Extracellular vesicles (EVs), including exosomes and microvesicles $(<200 \mathrm{~nm})$, play a vital role in intercellular communication and carry a net negative surface charge under physiological conditions. Zeta potential (ZP) is a popular method to measure the surface potential of EVs, while used as an indicator of surface charge, and colloidal stability influenced by surface chemistry, bioconjugation, and the theoretical model applied. Here, we investigated the effects of such factors on ZP of wellcharacterized EVs derived from the human choriocarcinoma JAr cells. The EVs were suspended in phosphate-buffered saline (PBS) of various phosphate ionic concentrations $(0.01,0.1$, and $1 \mathrm{mM})$, Zeta potential marks the colloidal stability in extracellular vesicles with or without detergent (Tween-20), or in the presence (10

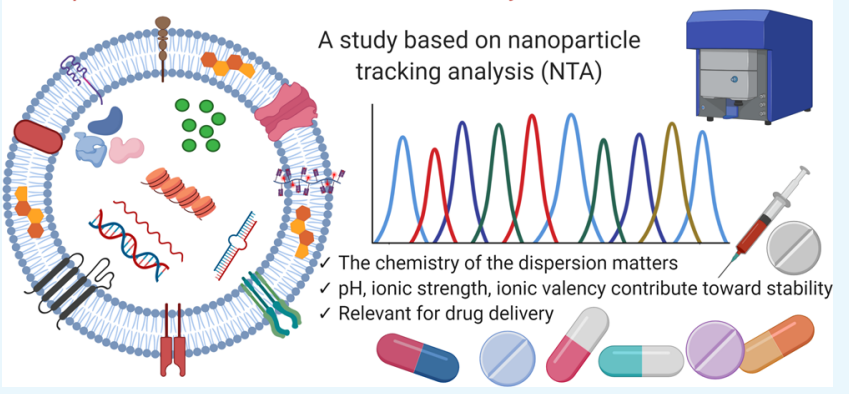
$\mathrm{mM})$ of different salts $\left(\mathrm{NaCl}, \mathrm{KCl}, \mathrm{CaCl}_{2}\right.$, and $\left.\mathrm{AlCl}_{3}\right)$ and at different $\mathrm{pH}$ values $(4,7$, and 10) while the $\mathrm{ZP}$ was measured. The $\mathrm{ZP}$ changed inversely with the buffer concentration, while Tween-20 caused a significant $(p<0.05)$ lowering of the ZP. Moreover, the $\mathrm{ZP}$ was significantly $(p<0.05)$ less negative in the presence of ions with higher valency $\left(\mathrm{Al}^{3+} / \mathrm{Ca}^{2+}\right)$ than in the presence of monovalent ones $\left(\mathrm{Na}^{+} / \mathrm{K}^{+}\right)$. Besides, the $\mathrm{ZP}$ of EVs became less negative at acidic $\mathrm{pH}$, and vice versa. The integrated data underpins the crucial role of physicochemical attributes that influence the colloidal stability of EVs.

\section{INTRODUCTION}

Research on extracellular vesicles (EVs), including exosomes and microvesicles, has witnessed a considerable boost over the past few years. EVs play a vital role in transporting functional molecules to the nearby or distant cells, thus facilitating intercellular communication (Figure 1). ${ }^{1}$ Such communication is not only essential for multicellular organisms but also being increasingly recognized to play a role in the pathogenesis of diseases. ${ }^{2}$ EVs are observed in biological fluids, such as serum, urine, and breast milk, while most cell types are capable of secreting exosomes under different conditions. ${ }^{3}$ Therefore, conditioned medium is a widely used source to isolate EVs for biological studies. The protein composition of exosomes is closely related to their biogenesis as well as the physiological conditions of the cells of origin. ${ }^{4}$ For instance, EVs are enriched in membrane proteins, endosome-specific proteins (Alix and TSG 101), tetraspanins (CD9, CD63, and CD81), and heat-shock proteins (HSPs, such as HSP70 and HSP90). ${ }^{5}$ Such surface proteins, in addition to the cargo of molecules present within the cores as payloads (e.g., RNA), have been posited to be a promising inventory of biomarkers, and, thus, exosomes may present a new possibility in oncotherapeutics in addition to the diagnosis and treatment of many other diseases. $^{6}$
Different analytical methods are applied for a better understanding of the EVs and their potential applications. ${ }^{8}$ However, it is challenging to analyze such heterogeneous nanoscale particles, suggesting a need for different platforms to characterize them. Traditionally, EVs have been characterized in terms of physical properties, such as particle size, concentration, surface charge, density, and biological properties, that is, their internal and external biomolecular composition, for instance, membrane-associated antigens. ${ }^{8,9}$ For measurements of particle size, shape, and density of EVs, techniques such as electron microscopy (EM) ${ }^{10}$ atomic force microscopy, ${ }^{11}$ dynamic light scattering (DLS), tunable resistive pulse sensing, ${ }^{12}$ flow cytometry, ${ }^{13}$ and nanoparticle tracking analysis (NTA) ${ }^{14,15}$ are used.

A thorough understanding of the in vivo interactions of EVs and their fate within the human body will improve their scope in nanomedicine. However, interactions between the particles

Received: April 7, 2020

Accepted: June 16, 2020

Published: June 30, 2020 


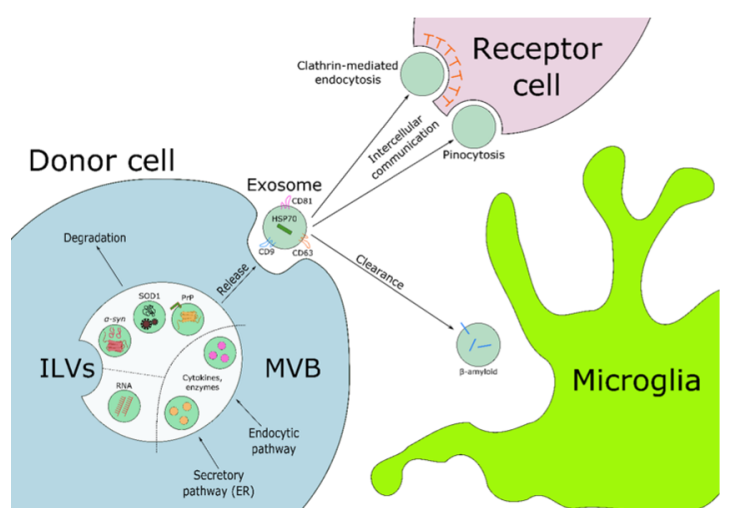

Figure 1. Physiology of exosomes: fusion of the multivesicular bodies (MVBs) with the cell membrane releases the exosomes decorated with markers such as CD9, CD63, CD81, and HSP70 and composed of intraluminal vesicles (ILVs) derived from the endoplasmic reticulum (ER) as part of the secretory and/or endocytic pathways. Exosomes carry nucleic acids, cytokines, and proteins ( $\alpha$-synuclein, superoxide dismutase/SOD1, $\operatorname{PrP}$ ) and achieve intercellular communication via a range of mechanisms, including uptake by the receptor cell by clathrin-mediated pathway or pinocytosis, while the toxic materials (e.g., $\beta$-amyloid) in the exosomes are cleared by the microglia and macrophages. Adapted as a freely available open access material under Creative Commons Attribution License (CC BY) from Soria et al., 2017.

in dispersed systems can be quite complex. Much of this complexity may arise from the differences in surface charge of the particles. As nanoparticles (NPs), nonfunctionalized EVs carry a net negative surface charge due to the nature of molecules expressed at their surfaces (Figure S1). Zeta potential (ZP), as an indicator of colloidal stability, is influenced by the surface charge and can be measured from the electrophoretic mobility in a suspension. Dispersed systems, such as emulsions, suspensions, and colloidal dispersions of NPs, contain electrically charged particles. In such dispersed systems, the net surface charge of NPs, as indicated by the $\mathrm{ZP}$, determines the stability of particleparticle and particle-medium interactions, including the tendency of the particles to aggregate. Therefore, $\mathrm{ZP}$ is one of the most useful tools to investigate the collective behavior of NPs, including colloidal stability, such as EVs in dispersed systems, and thus, holds promise as a method for studying the activity of EVs in biological processes. For example, the surface charge is known to influence different biological processes associated with NPs, such as cellular uptake ${ }^{16}$ and cytotoxicity. $^{17}$

According to the von Smoluchowski equation, the electrophoretic mobility of charged particles $\left(\mu_{\mathrm{e}}\right)$ is defined in terms of the physical properties of dispersion and ZP. ${ }^{18}$ However, depending on the allied factors, for example, particle size, surface charge, and Debye length, other electrokinetic theories, such as the Hückel, Henry, or O'Brien models, may be required to explain the $\mu_{\mathrm{e}}{ }^{19}$ Under physiological conditions, the surface of a biological plasma membrane carries a negatively charged network of glycosylated proteins intercalated within the lipid bilayer. ${ }^{20}$ EVs and the plasma membrane of cells possess a negative surface charge when suspended in a neutral medium. The surface charge of EVs depends on a multitude of factors: ionization of the membrane surface groups, the chemistry of grafted chains (if any), protonated states, inter- and intramolecular bonding, presence of $\mathrm{H}$-bonds, and ion adsorption from the electrolytes present in solution, to name a few. Hence, the magnitude of $\mathrm{ZP}$ can fluctuate depending on the electrochemical characteristics at the particle-medium interface and is affected by numerous factors, such as surface chemistry, $\mathrm{pH}$, and ionic strength of the medium, or the theoretical model applied. Although the effect of such factors on the ZP of synthetic and nonbiological NPs has been studied extensively, such investigations on biologically derived NPs, such as EVs, are rare. ${ }^{21}$ The effects of such factors on the ZP of EVs are unfortunately not characterized enough despite their crucial role in stability, pharmacokinetics, and release mechanism, which are essential topics for preparing nanomedicinal formulations. In this paper, we propose the $\mathrm{ZP}$ as an enabling characterization tool to unravel the complexities of $\mathrm{EV}$ interactions with their dispersant with tangible effects on colloidal stability and repercussions from applied aspects, such as for theranostic purposes. Thus, we aimed to investigate the effect of different factors on the ZP of EVs secreted by the cultured human choriocarcinoma (JAr) cells, while remaining focused primarily on physical properties that affect the ZP, such as the concentration of buffer, with or without surfactant, $\mathrm{pH}$ and, ionic strength. Such investigations are essential if $\mathrm{ZP}$ measurement protocols are to be reproducible, robust, and conducive to further uses of EVs as a drug delivery system (DDS).
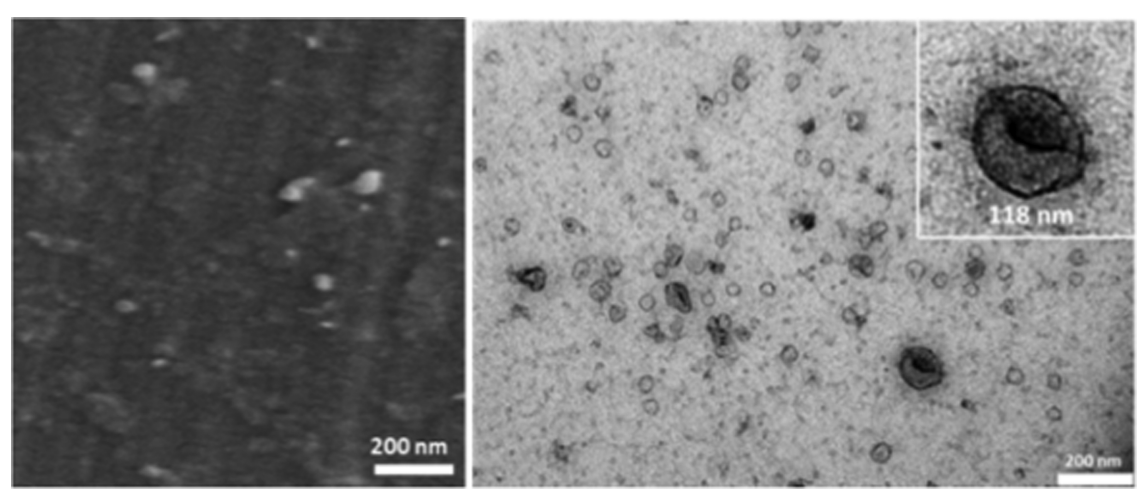

Figure 2. Morphology of JAr EVs was observed using SEM (left panel) and TEM (right panel, inset: zoomed view of an EV elucidating structural details). Scale bars are $200 \mathrm{~nm}$ for both the panels. 


\section{RESULTS AND DISCUSSION}

Interest in the EVs as a biologically derived nano-DDS has gained momentum due to their emerging importance in physiology and potential utility as theranostic agents. However, especially for formulation purposes, it is essential to gain control over the physicochemical attributes of such a nanoparticulate system that is inherently heterogeneous and lacks stability. It is established that the physicochemical properties, including particle size and surface charge, influence the way nanoscale materials behave and interact at biological interfaces. Understanding such complex interactions in a nanobio environment requires methods for accurate and standardized characterization of the $\mathrm{NPs},{ }^{22}$ which unfortunately continues to lack clarity and harmonization at a global scale. Similarly, the characterization of EVs, like many other NPs, has proved to be technically challenging due to a plethora of reasons, including their heterogeneity. ${ }^{23}$ In this study, we probed the role of various effects exerted by the dispersing medium on the $\mathrm{ZP}$ of EVs secreted by cultured human choriocarcinoma JAr cells. As a conditioned medium is a widely used source to isolate EVs for biological studies, we considered the use of JAr-cell-derived EVs to be apt for this project. It is also worth noting here that JAr cells are used to study trophoblast differentiation.

2.1. Characterization of the EVs. 2.1.1. Electron Microscopy and NTA Measurements. The morphology and size of the JAr EVs were determined by scanning electron microscopy (SEM) and transmission electron microscopy (TEM). Both the SEM and TEM images confirmed the overall spherical shape of the EVs (Figures 2, S2, and S3), while the mean diameter of the EVs $(\sim 120 \mathrm{~nm})$ matched the NTA data (Figure 3, Scheme S1, Videos S1 and S2), and a polydispersity

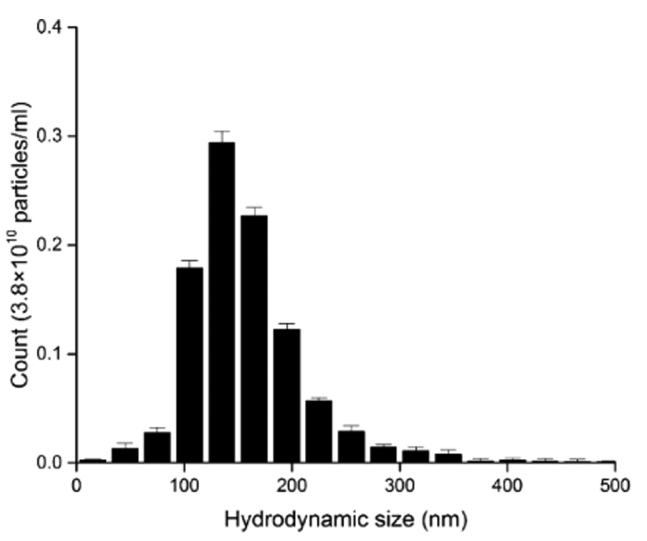

Figure 3. NTA-based hydrodynamic size distribution expressed as the mean \pm standard deviation $(\mathrm{SD})$ of the JAr EVs $(n=3)$. The PDI was calculated to be 1.11 .

index (PDI) of 1.11 was calculated (Scheme S2). Besides, the TEM data showed that the EVs were overall spherical, cupshaped, and heterogeneous in size. TEM analysis revealed that the size of EVs was $30-200 \mathrm{~nm}$ with a visible lipid bilayer (Figure 2, right panel).

The results of SEM and TEM confirmed the presence of EVs with a heterogeneity of size in the culture conditioned media of JAr cells. EVs with broad size distribution are more susceptible to lysis, ${ }^{24}$ and the present study investigated the crucial issue of colloidal stability in EV suspensions under physiologically relevant conditions and its vulnerability to a range of fluctuations, such as the $\mathrm{pH}$ and ionic strength. These factors are highly relevant to formulate EV-based nanomedical preparations in a controlled and reproducible manner, which has remained a less explored topic so far. Adequate separation between the various EV-subpopulations, that is, exosomes and microvesicles, is also necessary to allow individual $\mathrm{ZP}$ measurements. The use of NTA is better suited for EVsubpopulations as the measurements are done on a particle-byparticle basis. However, the introduction of another method, such as DLS, can be a useful verification tool for NTA results, although both the techniques have their strengths and weaknesses.

It is also important to note that the average hydrodynamic size of the detected EVs was dependent on the chosen software settings during NTA analysis. For example, our ZetaView measurement settings were optimized to detect small particles. As EVs are a heterogeneous mix of exosomes and microvesicles, it was possible to adjust the NTA settings inclined more toward detecting the larger particles, such as microvesicles, by decreasing the frame rate. Such nuances should be kept in mind during future research endeavors. Inclusion of data arising from the aggregated particles can also contaminate the particle size determined in NTA analysis ${ }^{25}$ and remains an issue.

2.1.2. Western Blot Analysis. Western blot analysis for EVspecific surface markers, such as CD9, CD63, CD81, and HSP70 (Figure 4), showed that the JAr EVs were positive for them.

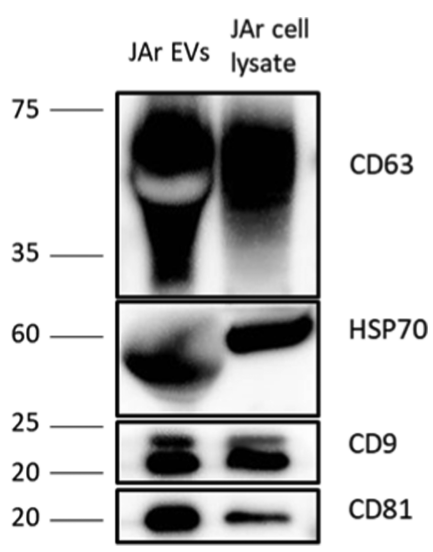

Figure 4. Western blot analysis with EV-specific markers: EVs isolated from the conditioned medium of human choriocarcinoma (JAr) cells were probed/immunoblotted with CD9, CD63, CD81, and HSP70.

The western blot analysis showed the presence of EVspecific surface markers, such as CD9, CD63, CD81, and HSP70-a cytosolic protein, both in the JAr EVs and cell lysates. The presence of a similar amount of these antigens was observed both in the EV preparations and cell lysates, indicating that the EVs were successfully isolated using size exclusion chromatography (SEC). The presence of such proteins on the surfaces decorated the EVs with anionic sites. However, it also rendered the EV surfaces more complex and challenging to exert control during their synthesis with defined surface chemistry or the way EVs interact with their biophysical surroundings. Furthermore, significant differences in ZP were observed for exosomes derived from human cell culture. $^{26-29}$ Therefore, additional research should be undertaken to investigate and compare the $\mathrm{ZP}$ data obtained under 
different conditions or using different instruments to assess the reported differences in such measurements on EVs. ${ }^{30}$

2.2. Effect of Buffer Content on the ZP of EVs. To investigate the effect of buffer content on the ZP of EVs, phosphate-buffered saline (PBS) was diluted to $1,0.1$, and 0.01 $\mathrm{mM}$ (phosphate) in water ( $\mathrm{pH}$ 6.9). Regardless of the EV concentration, the measured ZP showed a significant shift toward less negative values in the presence of higher concentrations of PBS $(F(2)=166.4, p<0.001$; Figure 5).

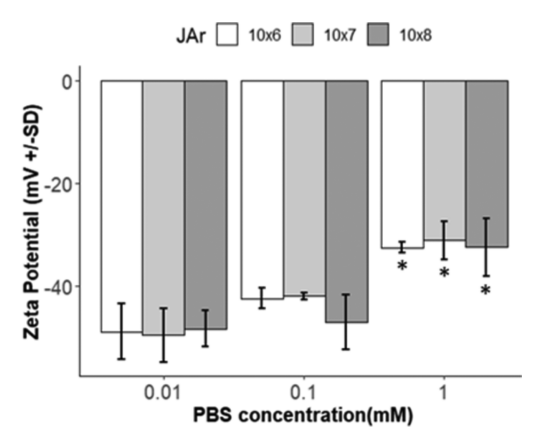

Figure 5. ZP of JAr EVs (mean $\pm \mathrm{SD}, n=9$ ) expressed as a function of different concentrations of EVs $\left(10^{6} / \mathrm{mL}(\square), 10^{7} / \mathrm{mL}\right.$ (light gray boxes), and $10^{8} / \mathrm{mL}$ (dark gray boxes)) and PBS (0.01, 0.1, and 1 $\mathrm{mM})$ at $\mathrm{pH} 6.9$. All of the data points at $1 \mathrm{mM}$ were significantly different $(p<0.05)$ than those for the most dilute, that is, $0.01 \mathrm{mM}$, dispersions carrying $0.138 \mathrm{M}$ and $0.0027 \mathrm{M} \mathrm{NaCl}$ and $\mathrm{KCl}$, respectively, and thus were marked with an asterisk $(*)$ symbol.

There was no significant effect of EV concentration $(F(2)=$ 1.04, $p=0.434)$ on the respective PBS concentrations. Post hoc Tukey's test revealed that for each of the EV concentrations, ZP was significantly less negative at $1 \mathrm{mM}$ compared to $0.01 \mathrm{mM}$ PBS (all $p<0.01$ ).

The state of dispersion can alter the surface chemistry of the NPs and, thereby, their surface charge. In this study, the ZP values of EVs became less negative as the PBS concentration increased. This effect can be attributed to the presence of a higher number of counterions in $1 \mathrm{mM}$ PBS compared to the two other $10 \times$ serial dilutions. The presence of counterions can be linked with the higher conductivity noticed at a higher molar concentration of PBS $(1 \mathrm{mM}[1070-1151 \mu \mathrm{S} / \mathrm{cm}]>$ $0.1 \mathrm{mM}[93-145 \mu \mathrm{S} / \mathrm{cm}]>0.01 \mathrm{mM}[26-46 \mu \mathrm{S} / \mathrm{cm}])$. Such high conductivity at $1 \mathrm{mM}$ PBS concentration may, thus, affect the ionic ambiance of the NPs. Specifically, the thickness of an electrical double layer (EDL) surrounding the particle has an inverse relationship with the ionic strength of the surrounding medium since an excess of ions can hold the EDL more robustly, hence causing shrinkage of the EDL. Additionally, a higher conductivity of the dispersing medium can influence the stability of EDL by lowering the repulsive forces between the EVs, which in turn augments the interparticulate interactions due to forces acting at a molecular scale, such as the Van der Waals force; thus interfering with the stability of the colloidal system of EVs and reflected in a less negative ZP value. Our results corroborated well with some of the previous studies conducted under similar lines. ${ }^{26,27}$ However, contrary to our findings, a previous study showed that decreasing molar concentrations of PBS led to less negative $\mathrm{ZP}$ of normal trabecular meshwork-derived exosomes. ${ }^{31}$ Such differences in observation might have appeared due to several factors, such as the range of PBS concentrations tested, type of EVs studied along with particle concentration, dispersing medium for the formation of EV-complexes, etc. Overall, the dependence of ZP on EV concentration was observed in each of the PBS concentrations.

Nevertheless, the results indicated that there was a concentration range where the $\mathrm{ZP}$ was affected by the particulate concentrations of EVs suspended in $0.1 \mathrm{mM}$ PBS buffer content. The two lower concentrations of EVs $\left(10^{6} / \mathrm{mL}\right.$ and $10^{7} / \mathrm{mL}$ ) showed a shift in $\mathrm{ZP}$ to less negative values compared to the highest concentration of EVs $\left(10^{8} / \mathrm{mL}\right)$ resuspended in the same buffer content. The shift in $\mathrm{ZP}$ values at lower concentrations may be accompanied by the presence of higher electrophoretic mobility of EVs in dispersing medium and low scattering signals. In other words, NPs were too diluted at lower concentrations and, hence, the particles failed to achieve a satisfactory signal-to-noise ratio. Thus, the net effect on ZP may change with the concentration of NPs and dispersing medium. Therefore, it is advisable to limit aggregation by decreasing the NP concentration up to an optimal standard that still delivers an adequate signal-to-noise ratio. In general, it is difficult to provide a relationship between $\mathrm{ZP}$ and NP concentration. Additionally, osmotic effects due to the varying ionic strength can induce swelling or shrinkage of the EVs, which in turn can also affect ZP.

2.3. Effect of Detergent on the ZP of EVs. The addition of $0.03 \%$ Tween-20 induced a shift toward less negative ZP values, which appeared to be overall consistent across the different EV and PBS concentrations (Figure 6). As such, there

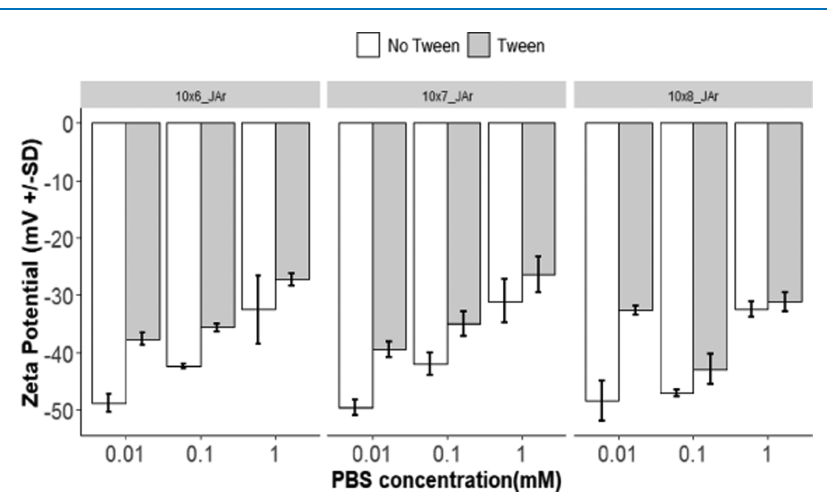

Figure 6. ZP (mean $\pm \mathrm{SD}, n=9$ ) of JAr EVs under different buffer conditions. The EVs were suspended in PBS of various concentrations $(0.01,0.1$, and $1 \mathrm{mM})$, without (gray boxes) or with $(\square)$ Tween-20 $(0.03 \%)$, at $\mathrm{pH} 6.9$ under three different particle concentrations $\left(10^{6} /\right.$ $\mathrm{mL}, 10^{7} / \mathrm{mL}$, and $10^{8} / \mathrm{mL}$ ).

was a highly significant additive effect of Tween $(F(2)=$ 279.64, $p=0.004)$. The effect of Tween was significantly dependent on buffer concentration $(F(2)=10.2, p=0.027)$ but not EV concentration $(F(2)=0.442, p=0.67)$. Post hoc Tukey's test showed the effect of Tween to be significant $(p<$ $0.05)$ at all PBS dilutions and EV concentrations except the measurements at $1 \mathrm{mM} \mathrm{PBS}$ and $10^{8} / \mathrm{mL}(p=0.4)$ concentration.

The present study was also designed to determine the effect of detergents on the ZP of EVs. To assess possible interactions of complex adsorbates at the solid-liquid and liquid-liquid interfaces, we used Tween-20, a widely used non-ionic detergent, to determine the degree of adsorption onto the EVs. The addition of detergent to the buffer solutions led to less negative ZP values. In general, EVs suspended in PBS and supplemented with $0.03 \%$ Tween-20 showed less negative $\mathrm{ZP}$, 
which was more apparent at a higher molar concentration of PBS. However, the presence of Tween-20 did not affect the ZP for all of the EV and buffer combinations. For example, there was hardly any effect of Tween-20 on ZP measurements in 1 $\mathrm{mM}$ PBS at the highest EV concentration $\left(10^{8} / \mathrm{mL}\right)$. It is known that non-ionic detergents or surfactants are capable of breaking lipid-lipid and lipid-protein interactions more than protein-protein interactions and rarely denature proteins. ${ }^{32}$ Such surfactants are used to control the surface charge and/or the hydrophobic-hydrophilic balance at particle surfaces, thus influencing the colloidal stability. Here, the addition of a nonionic detergent into PBS presumably led to its adsorption on the EVs and deterred colloidal stability. The further reduction in the ZP of EVs was likely caused by the ability of the surfactant to be adsorbed onto EV surfaces/lipid bilayer near the critical micellar concentration (CMC). Moreover, it is possible that the presence of free fatty acids (FFAs), which are known to interact with Tween-20, may impart a negative ZP. Overall, the ZP of EVs in the suspending medium might have stabilized due to non-ionic interactions of detergent in the presence of low molar concentrations of PBS interfering with the steric repulsion between EVs. Although a study showed the effect of detergents on the susceptibility of EV lysis, ${ }^{24}$ no previous study investigated the effect of detergents on ZP or its correlation to the colloidal stability of EVs.

2.4. Effect of Ionic Valency and lonic Strength on the ZP of EVs. The ZP of EVs became less negative in the presence of trivalent $\mathrm{Al}^{3+}$ (mean $\mathrm{ZP},-33 \mathrm{mV} ; \mathrm{T}(8)=13.52$, $p$ $<0.001$ ) and divalent $\mathrm{Ca}^{2+}$ (mean $\mathrm{ZP},-24 \mathrm{mV} ; \mathrm{T}(8)=9.86, p$ $<0.001$ ) but was not affected by the monovalent $\mathrm{Na}^{+}$and $\mathrm{K}^{+}$ ions (Figure 7 , left panel). The $\mathrm{ZP}$ value decreased with increasing ionic strength (Figure 7, right panel).

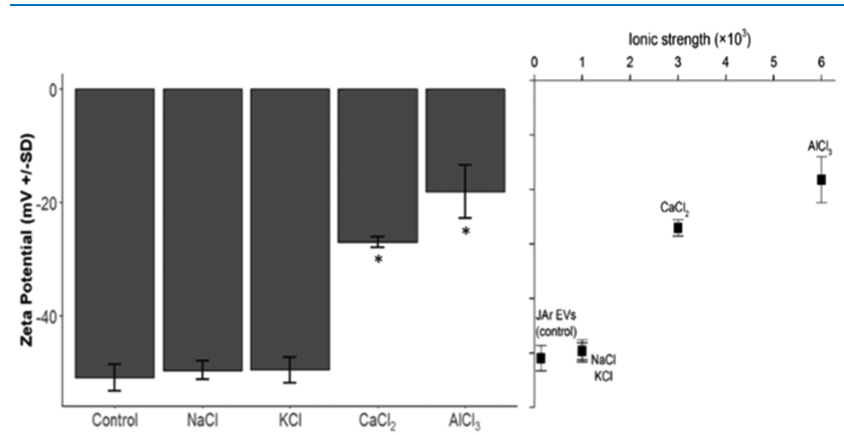

Figure 7. ZP (mean $\pm \mathrm{SD}, n=9$ ) of JAr EVs: (left panel) in the presence of $10 \mathrm{mM}$ different chloride salts $\left(\mathrm{NaCl}, \mathrm{KCl}, \mathrm{CaCl}_{2}\right.$, and $\mathrm{AlCl}_{3}$ ) in $0.01 \mathrm{mM} \mathrm{PBS}(\mathrm{pH} \mathrm{6.9)}$ and (right panel) at various ionic strengths. The $\mathrm{ZP}$ values in the presence of $\mathrm{CaCl}_{2}$ and $\mathrm{AlCl}_{3}$ (ionic strength $\left.6 \times 10^{3}\right)$ were significantly different $(p<0.05)$ than the control and, thus, were marked with an asterisk (*) symbol.

It is worth mentioning here that apart from $\mathrm{ZP}$, the critical coagulation concentration (CCC), that is, the minimum concentration of the counterions required to induce coagulation in a colloidal suspension, may also be a crucial parameter to assess colloidal stability. The CCC values may be calculated not only based on the energy ${ }^{33}$ or force ${ }^{34}$ of the interactions occurring within a dispersion but also from the electrophoretic mobility. ${ }^{35}$ Furthermore, the factors that influence the $\mathrm{ZP}$ and included in this study might provide with essential cues on the fate of the EVs within cellular cytoplasm known to undergo fluctuations regarding their ionic strength and $\mathrm{pH}^{36}$
The ZP of JAr EVs became significantly less negative in the presence of trivalent ions compared to the monovalent or divalent ones. The decrease in ZP magnitude in response to higher-valency ions is likely due to the compression of the EDL surrounding EV surfaces resulting from an increased binding by the ions of higher valency. Thus, the magnitude of $\mathrm{ZP}$, or the colloidal stability, of JAr EVs at neutral $\mathrm{pH}$ decreased in the following order: $\mathrm{Al}^{3+}>\mathrm{Ca}^{2+}>\mathrm{Na}^{+}, \mathrm{K}^{+}$. According to the DLVO (Derjaguin, Landau, Verwey, and Overbeek) model, the interaction between the particles in a system is the sum of the attractive and repulsive forces. If the net repulsive forces exceed the net attractive forces, the colloidal system gains stability as it deters close contact within the particles, ${ }^{37}$ and vice versa. Unfortunately, for the monovalent ions, such as the $\mathrm{N}^{+}$ and $\mathrm{K}^{+}$, the DLVO model fails to predict the CCC accurately due to its dependence on valency and concentration of ions present in the dispersion. In reality, though, the CCC depends on multiple factors, including the properties of co-ions and counterions. Therefore, the Hofmeister series provides a better prediction of colloidal stability than the DLVO model in case of monovalent ions. ${ }^{38}$ For multivalent ions, however, the DLVO model predicts the CCC as per the Schulze-Hardy rule, which states that an ion causing coagulation in a colloidal dispersion carries an opposite charge to the colloidal particles and its coagulating power increases with valency. ${ }^{39}$ The ZP of JAr EVs became less negative with increasing valency of ions that compressed the EDL and, thus, facilitated the proximity between the particles, resulting in aggregation, as reflected in less negative ZP. The presence of monovalent ions $\mathrm{Na}^{+}, \mathrm{K}^{+}$, and $\mathrm{Cl}^{-}$did not affect the measured $\mathrm{ZP}$ of EVs. Although some similar studies based on the effect of proteins are available, ${ }^{40}$ to the best of our knowledge, this study is the first to highlight the role of ionic valency in the colloidal stability of EV dispersions.

2.5. Effect of $\mathrm{pH}$ on the ZP of EVs. Overall, ZP became more negative as $\mathrm{pH}$ increased $(F(2)=21.66, p=0.007$; Figure 8$)$ but appeared to be more variable at alkaline and

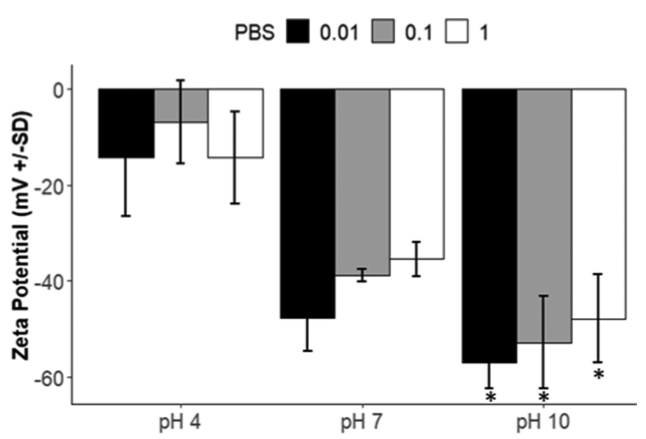

Figure 8. ZP measurements (mean $\pm \mathrm{SD}, n=9$ ) of $10^{8} \mathrm{EVs} / \mathrm{mL}$ expressed as a function of $\mathrm{pH}$ (acidic $\mathrm{pH} 4$, neutral $\mathrm{pH} 7$, and basic $\mathrm{pH} 10$ ) in the presence of $0.01 \mathrm{mM}(\boldsymbol{\square}), 0.1 \mathrm{mM}$ (gray boxes), and 1 $\mathrm{mM}(\square)$ PBS. All of the data points at $1 \mathrm{mM}$ were significantly different $(p<0.05)$ than those of the most dilute, that is, $0.01 \mathrm{mM}$, dispersions and thus were marked with asterisk $(*)$ symbols.

acidic $\mathrm{pH}$ values than at neutral $\mathrm{pH}$ 7. Post hoc Tukey's tests showed that $\mathrm{ZP}$ was significantly more negative at $\mathrm{pH} 10$ compared to $\mathrm{pH} 4$ at all PBS concentrations (all $p<0.01$ ). The effect of $\mathrm{pH}$ was not influenced by the buffer content, though $(F(4)=1.45, p=0.304)$.

Our study showed the effect of $\mathrm{pH}$ on the ZP of EVs: the ZP became less negative at acidic $\mathrm{pH}$ with more propensity toward 
aggregation, and vice versa. One hypothesis is that primarily due to the presence of lipids and proteins, including the CD antigens, EVs harbor ample anionic sites on their surfaces, resulting in a negative $\mathrm{ZP}$ with a magnitude of $30-40 \mathrm{mV}$. However, the presence of excess protons $\left(\mathrm{H}^{+}\right)$under acidic conditions most likely neutralized some of the anionic sites on EV surfaces and compromised the electrostatic interparticulate repulsive forces to destabilize the system, as got noticed in lesser negative ZP values. In contrast, the presence of an excess base under basic conditions unmasked additional negative sites on the EV surfaces by deprotonation of the surface groups, thus adding to the interparticulate repulsive forces, and enhanced colloidal stability reflected by more negative ZP values. Some studies have reported the effect of multiple storage conditions, such as the $\mathrm{pH}$, particle concentration, ${ }^{41}$ EV isolation, ${ }^{42}$ and overall colloidal stability. Our data provided a conceptual framework to explain such findings, prioritized physicochemical characterization for a successful translational of the EVs, and informed researchers in the field to ascertain potential risks associated with the nature of EVs.

\section{CONCLUSIONS}

This study aimed to check the applicability of ZP for the direct characterization of EVs. We used EVs secreted from the human choriocarcinoma JAr cells as a model system. Higher concentrations of multivalent ions, both cationic $\left(\mathrm{Ca}^{2+}, \mathrm{Al}^{3+}\right)$ and anionic (phosphate), decreased the negative value of $\mathrm{ZP}$ at all EV concentrations $\left(10^{6} / \mathrm{mL}, 10^{7} / \mathrm{mL}\right.$, and $\left.10^{8} / \mathrm{mL}\right)$ investigated. The influence of monovalent ions on $\mathrm{ZP}$ values at their concentrations up to $10 \mathrm{mM}\left(\mathrm{K}^{+}, \mathrm{Na}^{+}\right.$, and $\left.\mathrm{Cl}^{-}\right)$was minimal. The ZP of the studied EVs was dependent on the $\mathrm{pH}$ of the solution. Besides, the presence of detergent (Tween-20) considerably decreased the negative value of $\mathrm{ZP}$.

Our data indicated a need for the characterization of EVs under standardized ZP measurement protocols. Without such protocols, or reporting the precise experimental conditions used while measuring $\mathrm{ZP}$, the obtained data will be irreproducible and hardly bear any value from a translational perspective or characterization of the EVs.

\section{EXPERIMENTAL SECTION}

4.1. Materials. Dulbecco's PBS (Sigma-Aldrich Co., St. Louis), Tween-20 (0.0003\% Arsenic, 0.0005\% Copper, Fisher BioReagents, Fair Lawn), hydrochloric acid (Honeywell $\mathrm{GmbH}$, Seelze, Germany), sodium hydroxide (potassium $\leq 0.02 \%, \geq 98 \%$, Sigma-Aldrich Co., St. Louis), sodium chloride (Merck KGaA, Darmstadt, Germany), potassium chloride ( $\geq 99 \%$, Fisher Chemical, Loughborough, U.K.), calcium chloride ( $\geq 93 \%$, Sigma-Aldrich Co., St. Louis), and aluminum chloride ( $\geq 99 \%$, Chem-Supply Pty Ltd, Gillman, Australia) were used as received without any further purification.

4.2. Cell Culture. The human choriocarcinoma cell line (JAr) from the first trimester trophoblasts was acquired from the American Type Culture Collection (ATCC, HTB-144, Teddington, U.K.). JAr cells were cultured in a T75 flask in Roswell Park Memorial Institute (RPMI) 1640 media (Gibco, Scotland) supplemented with $1 \%$ penicillin/streptomycin (P/ S, Gibco 15140122, Bleiswijk, Netherlands), 1\% L-glutamine (Sigma, 59202C, St. Louis), and 10\% fetal bovine serum (FBS, Gibco, 10500064) at $37{ }^{\circ} \mathrm{C}$ under moist $5 \% \mathrm{CO}_{2}$-rich conditions. At $80 \%$ confluence, the conditioned medium was removed, and the cells were washed with $10 \mathrm{~mL}$ of nonsupplemented RPMI 1640 media to remove traces of FBS. Nonsupplemented RPMI 1640 medium was replaced with fresh RPMI 1640 medium supplemented with $1 \%$ penicillin/streptomycin, 1\% L-glutamine, and 10\% EV-depleted FBS. Cells were cultured for $24 \mathrm{~h}$ at $37^{\circ} \mathrm{C}$ and $5 \% \mathrm{CO}_{2}$. After incubation, the conditioned medium was collected for $\mathrm{EV}$ isolation. We have submitted all relevant data of our experiments to the EV-TRACK knowledgebase (EV-TRACK ID: EV190091). ${ }^{43}$

4.2.1. EV-Depletion of the FBS. The depletion of EVs in FBS was carried out using a methodology proposed earlier. ${ }^{44}$ Briefly, FBS was ultrafiltered using Amicon Ultra- 15 centrifugal filter devices (100 kDa cutoff, MerkMillipore, Darmstadt, Germany) for $30 \mathrm{~min}$ at $5000 \mathrm{~g}$. The filtrate was collected and measured for particle concentration using NTA. For this study, the vesicle-depleted conditioned medium was used to isolate and purify EVs by SEC, and the EV preparations met the optimal quality as per the guidelines prescribed by the International Society for Extracellular Vesicles (ISEV). ${ }^{45}$

4.2.2. EV-Purification. The conditioned medium was centrifuged first at $400 \mathrm{~g}$ for $10 \mathrm{~min}$, while the supernatant from two successive centrifugation steps $(4000 \mathrm{~g}$ and $10000 \mathrm{~g}$ for $10 \mathrm{~min}$ ) to remove cell debris and apoptotic bodies was retained. The collected conditioned medium was concentrated to $500 \mu \mathrm{L}$ with Amicon Ultra-15 centrifugal filter devices (10 kDa cutoff, MerkMillipore, Darmstadt, Germany), and EVs were isolated from the concentrated media using SEC in a cross-linked $4 \%$ agarose matrix of $90 \mu \mathrm{m}$ beads (Sepharose 4 Fast Flow, GE HealthCare Bio-Sciences AB, Uppsala, Sweden) in a $10 \mathrm{~cm}$ gravity column calibrated with PBS. The EVenriched fractions 6-9 (volume $0.5 \mathrm{~mL}$ ) were collected and concentrated further using Amicon Ultra-15 centrifugal filter devices (10 kDa cutoff), as described previously. ${ }^{46}$ Isolated EVs were quantified using NTA (ZetaView, Particle Metrix $\mathrm{GmbH}$, Inning am Ammersee, Germany).

4.3. NTA on the EVs. NTA was conducted using a ZetaView PMX 110 V3.0 instrument (Particle Metrix GmbH, Germany), while the data were analyzed by ZetaView NTA software. Operating instructions of the manufacturer were followed before calibrating the instrument with a known concentration of $100 \mathrm{~nm}$ polystyrene nanoparticles (Applied Microspheres B.V., Netherlands). The standards were suspended in particle-free water, whereas the EV samples were diluted 1:1000 with Milli-Q (MQ) water for analyses. Particles were counted and size-distributed at three cycles of 11 frames per cycle under a sensitivity of 85 and shutter value of 100 .

4.4. ZP Measurements. The ZP of JAr EVs was measured thrice at $25{ }^{\circ} \mathrm{C}$ under the following settings: sensitivity of 85 , a shutter value of 70 , and a frame rate of 30 frames per second, while ZetaView software was used to collect and analyze the data.

4.5. SEM. A drop of the JAr EV suspension was deposited on an aluminum foil for overnight drying and imaged the following day in a Hitachi S-4300 SEM microscope after sputter-coating the samples with gold.

4.6. TEM. JAr EVs for TEM imaging were prepared as described before ${ }^{46}$ with modifications. Briefly, $20 \mu \mathrm{L}$ of the EV suspension was deposited on formvar-carbon-coated 200 mesh copper grids (Agar Scientific, Essex, U.K.) for $20 \mathrm{~min}$ and fixed in Karnowski fixatives (a mixture of $2 \%$ paraformaldehyde and $1 \%$ glutaraldehyde) for $5 \mathrm{~min}$. Next, EVs were contrasted for 5 min in uranyl oxalate [1:1 mixture of $4 \%$ uranyl acetate 
(21447-25, Polysciences, Warrington) and 0.15 M oxalic acid (75688, Sigma-Aldrich, Schnelldorf, Germany)] and embedded in a mixture (9:1) of $2 \%$ methylcellulose (M6385, SigmaAldrich, Schnelldorf, Germany) and 4\% uranyl acetate (2144725, Polysciences, Warrington). The EVs were imaged using a JEM 1400 TEM (JEOL Ltd. Tokyo, Japan, with Morada TEM CCD camera, Olympus, Germany) at $80 \mathrm{kV}$.

4.7. Western Blot Analysis. Purified JAr EVs were concentrated to $200 \mu \mathrm{L}$ with an Amicon Ultra-15 centrifugal filter unit (10 kDa cutoff, Millipore, Merck KGaA, Darmstadt, Germany). The concentrated sample was then transferred to a fresh tube, and EV-proteins were precipitated by adding 200 $\mu \mathrm{L}$ of water, $400 \mu \mathrm{L}$ of methanol, and $100 \mu \mathrm{L}$ of chloroform. The solution was briefly vortexed and centrifuged at $14000 \mathrm{~g}$ for $5 \mathrm{~min}$ at room temperature. Proteins formed a white precipitate at the interphase of the top aqueous layer. After removing the top layer, another $400 \mu \mathrm{L}$ of methanol was added to the precipitated proteins and was centrifuged again. The pellets were air-dried and resuspended in $0.5 \%$ sodium dodecyl sulfate (SDS), and protein concentrations were determined by the Bradford assay. JAr cells were lysed in lysis buffer $(50 \mathrm{mM}$ Tris- $\mathrm{HCl} \mathrm{pH} 7.5,150 \mathrm{mM} \mathrm{NaCl}, 1 \%$ Triton X-100, and $1 \times$ protease inhibitors) on ice for an hour. Samples were centrifuged at $14000 \mathrm{~g}$ for $5 \mathrm{~min}$ at $4{ }^{\circ} \mathrm{C}$, and the protein concentration in supernatants was determined by the Bradford assay. Either reducing or nonreducing Laemmli buffer was added to the protein samples and heated for $5 \mathrm{~min}$ at $95^{\circ} \mathrm{C}$. Then, $50 \mu \mathrm{g}$ of protein was used for the detection of HSP70 (under reducing conditions), and $30 \mu \mathrm{g}$ of protein was used for the detection of CD63, CD9, and CD81 (under nonreducing conditions). Protein samples were resolved in $12 \%$ SDSpolyacrylamide gel electrophoresis (PAGE) according to the standard protocol and transferred onto poly(vinylidene difluoride) membranes (Thermo Scientific, Michigan). The membrane was blocked in 5\% nonfatty dry milk in PBS-T (0.05\% Tween-20, Thermo Scientific, Michigan) for $1 \mathrm{~h}$ at room temperature followed by incubation with the primary anti-CD63 (556019, 1:1000, BD Biosciences, New Jersey), anti-CD9 (sc-59140, 1:250, Santa Cruz Biotechnology Inc., Dallas, TX), anti-HSP70 (NB110-61582, 1:1000, Novus Biologicals, Abington, U.K.), and anti-CD81 (555675, 1:500, BD Biosciences, New Jersey) antibodies overnight at $4{ }^{\circ} \mathrm{C}$ in $5 \%$ milk-PBS-T solution. Incubation with the horseradish peroxidase-conjugated goat anti-mouse secondary antibody (G21040, 1:10 000, Thermo Fisher Scientific, Eugene) was performed at room temperature for an hour. In between incubations, the membrane was washed thrice for $5 \mathrm{~min}$ in PBS-T. Protein bands were detected using the ECL SelectTM western blotting detection reagent (GE Healthcare, Buckinghamshire, U.K.) with an ImageQuantTM RT ECL imager (GE Healthcare, Buckinghamshire, U.K.).

4.8. Statistical Analysis. Statistical analyses were performed using $\mathrm{R}$ (version 3.6.1, $\mathrm{R}$ foundation for statistical computing, Vienna, Austria). The effects of different conditions were assessed using nested one-way or two-way ANOVAs with a "replicate" error term included. Tukey's multiple comparison tests were used for specific intergroup comparisons ("emmeans package"). Data are presented as mean $\pm \mathrm{SD}(n=3)$, while $p$-values $<0.05$ were taken as statistically significant and were marked with an asterisk (*) symbol. The data were plotted using the ggplot2 package in $\mathrm{R}$ and OriginPro 2015 software (OriginLab Corp., MA).
4.9. Experimental Design. 4.9.1. Effect of Buffer Concentration on the ZP of EVs. To study the effect of PBS concentration on the ZP of EVs, $20 \mu \mathrm{L}$ of the JAr EVs was suspended in a final volume of $200 \mu \mathrm{L}$ of PBS diluted in MQ water in a ratio of $1: 1000$ to obtain an initial concentration in the range of $10^{9}$ particles $/ \mathrm{mL}$. ZetaView NTA was used to measure the hydrodynamic size profile and concentration of JAr EVs suspended in 1:10 and 1:100 diluted PBS/MQ. Using such PBS dilutions (1, 0.1, and $0.01 \mathrm{mM})$, samples with diverse $\mathrm{EV}$ concentrations, ranging from $10^{6}$ to $10^{8} \mathrm{EVs} / \mathrm{mL}$, were prepared and incubated at $4{ }^{\circ} \mathrm{C}$ for $3 \mathrm{~h}$ on a BIOSAN Multi Bio RS-24 programmable rotator (BioSan, Riga, Latvia) set at $30 \mathrm{rpm}$. After the incubation, the $\mathrm{pH}$ of the suspension medium was adjusted to 6.9 with a $\mathrm{pH}$ meter (SevenCompact $^{\mathrm{TM}} \mathrm{pH} /$ Ion S220, Mettler-Toledo AG, Schwerzenbach, Switzerland) by addition of $0.1 \mathrm{M} \mathrm{HCl} / \mathrm{NaOH}$. The ZP of JAr EV samples was measured using ZetaView software. The experiments were performed on 3 days, and on each day, triplicates of $\mathrm{ZP}$ measurements were taken for individual samples with different concentrations of JAr EVs.

4.9.2. Effect of the Presence of Detergent on the ZP of $E V s$. To test whether the presence of detergent in the PBS buffer affected the ZP of EVs, $20 \mu \mathrm{L}$ of the JAr EVs was suspended in a final volume of $200 \mu \mathrm{L}$ of $1: 1000$ diluted in PBS/MQ containing 0.03\% Tween-20 (BP337, Fisher BioReagents, Fair Lawn) to obtain an initial concentration in the range of $10^{9}$ particles $/ \mathrm{mL}$. JAr EVs were also suspended in PBS diluted in $0.03 \%$ Tween-20/MQ in the ratios of $1: 10$ and $1: 100$. Using the same PBS concentrations (1, 0.1, and 0.01 $\mathrm{mM}$ ), samples with different EV concentrations ranging from $10^{6}$ to $10^{8} \mathrm{EVs} / \mathrm{mL}$ were prepared and incubated further at 4 ${ }^{\circ} \mathrm{C}$ for $3 \mathrm{~h}$ on a BIOSAN Multi Bio RS-24 rotator. After incubation, the $\mathrm{pH}$ of the suspension medium was adjusted to 6.9. The experiments were performed on 3 days and each day with triplicates of ZP measurements performed for each concentration of JAr EVs.

4.9.3. Effect of Ionic Strength of the Media on the ZP of $E V s$. Different chloride salts were used to test the effect of ionic strength on the ZP of EVs. For both the control and test samples, JAr EV suspensions, with an initial concentration of $10^{9} \mathrm{EVs} / \mathrm{mL}$ in PBS diluted in MQ at the ratio of $1: 1000$ to obtain a final concentration in the range of $10^{8} \mathrm{EVs} / \mathrm{mL}$, were used. Subsequently, $20 \mu \mathrm{L}$ of JAr EVs $\left(10^{9}\right.$ particles $\left./ \mathrm{mL}\right)$ was resuspended in $200 \mu \mathrm{L}$ of PBS/MQ in a ratio of $1: 1000$ and incubated at room temperature for an hour on a BIOSAN Multi Bio RS-24 rotator. For the test samples, the following chloride salts were used: $\mathrm{NaCl}, \mathrm{KCl}, \mathrm{CaCl}_{2}$, and $\mathrm{AlCl}_{3}$, with increasing valency of the cations, that is, monovalent sodium and potassium, divalent calcium, and trivalent aluminum, while a final concentration of $10 \mathrm{mM}$ was added separately into the EVs suspended in PBS/MQ (1:1000). Both the control and test samples had a final concentration in the range of $10^{8}$ particles/mL. After the incubation, the $\mathrm{pH}$ of the suspension was adjusted to 6.9 , using $0.1 \mathrm{M} \mathrm{HCl} / \mathrm{NaOH}$. The EVs were appropriately diluted in the respective buffer to fit into a predefined limit of the minimum number of detected particles (at least 100 particles/image frame). Later, the ZP of JAr EVs, with or without the presence of cations, was measured as described before in the paper.

4.9.4. Effect of $p H$ on the ZP Measurements of the EV. To study the effect of $\mathrm{pH}$ on the $\mathrm{ZP}, 10 \mu \mathrm{L}$ of JAr EVs $(\sim 2.2 \times$ $10^{10}$ particles $/ \mathrm{mL}$ ) was suspended in a final volume of $1000 \mu \mathrm{L}$ of PBS diluted in MQ at a ratio of 1:1000 to obtain a 
concentration in the range of $10^{8}$ particles $/ \mathrm{mL}$ during incubation. ZetaView was used to conduct the hydrodynamic size profiling and determination of the concentration of JAr EVs. Similarly, JAr EVs were also suspended in PBS diluted in MQ at the ratios of $1: 10$ and 1:100. Using such PBS concentrations $(1,0.1$, and $0.01 \mathrm{mM})$, samples with the same $\mathrm{EV}$ concentration of $10^{8} \mathrm{EVs} / \mathrm{mL}$ were prepared and incubated at $4{ }^{\circ} \mathrm{C}$ for $3 \mathrm{~h}$ on a BIOSAN Multi Bio RS-24 rotator. After the incubation, the $\mathrm{pH}$ of the suspension was adjusted to 4,7 , and 10 using a $\mathrm{pH}$ meter with the addition of $0.1 \mathrm{M} \mathrm{HCl} /$ $\mathrm{NaOH}$. EVs were diluted in the respective buffer to fit into a predefined limit of a minimum number of detected particles in the NTA ZetaView instrument, and the ZP of the EVs was measured as mentioned above. The experiments were performed on 3 days and each day in triplicates for each concentration of EVs.

\section{ASSOCIATED CONTENT}

\section{(s) Supporting Information}

The Supporting Information is available free of charge at https://pubs.acs.org/doi/10.1021/acsomega.0c01582.

Cartoon showing the molecules in an EV that impart the negative charge under a physiological $\mathrm{pH}$ of 7.4, SEM images of the EVs, TEM images of the EVs, and calculation of the PDI (PDF)

NTA data on JAr EVs in $10 \mathrm{mM} \mathrm{CaCl}_{2}$ solution (AVI) NTA data on JAr EVs in pH 4 (AVI)

\section{AUTHOR INFORMATION}

\section{Corresponding Author}

Alireza Fazeli - Department of Pathophysiology, Institute of Biomedicine and Translational Medicine, University of Tartu, Tartu, Estonia; Academic Unit of Reproductive and Developmental Medicine, Department of Oncology and Metabolism, Medical School, University of Sheffield, Sheffield, U.K.; 우 orcid.org/0000-0003-0870-9914;

Email: alireza.fazeli@ut.ee

\section{Authors}

Getnet Midekessa - Department of Pathophysiology, Institute of Biomedicine and Translational Medicine, University of Tartu, Tartu, Estonia

Kasun Godakumara - Department of Pathophysiology, Institute of Biomedicine and Translational Medicine, University of Tartu, Tartu, Estonia

James Ord - Department of Pathophysiology, Institute of Biomedicine and Translational Medicine, University of Tartu, Tartu, Estonia

Janeli Viil - Department of Pathophysiology, Institute of Biomedicine and Translational Medicine, University of Tartu, Tartu, Estonia

Freddy Lättekivi - Department of Pathophysiology, Institute of Biomedicine and Translational Medicine, University of Tartu, Tartu, Estonia

Keerthie Dissanayake - Department of Pathophysiology, Institute of Biomedicine and Translational Medicine, University of Tartu, Tartu, Estonia

Sergei Kopanchuk - Institute of Chemistry, University of Tartu, Tartu, Estonia

Ago Rinken - Institute of Chemistry, University of Tartu, Tartu, Estonia; 이이.orid.org/0000-0002-7238-749X
Aneta Andronowska - Department of Hormonal Action Mechanisms, Institute of Animal Reproduction and Food Research, Polish Academy of Sciences, Olsztyn, Poland

Sourav Bhattacharjee - School of Veterinary Medicine, University College Dublin, Dublin, Ireland; ํㅏ이이.org/00000002-6528-6877

Toonika Rinken - Institute of Chemistry, University of Tartu, Tartu, Estonia

Complete contact information is available at:

https://pubs.acs.org/10.1021/acsomega.0c01582

\section{Notes}

The authors declare no competing financial interest.

\section{ACKNOWLEDGMENTS}

This work was supported by the European Union's Horizon 2020 research and innovation program under grant agreement no. 668989. S.B. would like to acknowledge UCD Research for funding and Prof. Dimitri Scholz (UCD Conway Institute) for assistance while acquiring the SEM images.

\section{REFERENCES}

(1) Jella, K. K.; Nasti, T. H.; Li, Z. T.; Malla, S. R.; Buchwald, Z. S.; Khan, M. K. Exosomes, their biogenesis and role in inter-cellular communication, tumor microenvironment and cancer immunotherapy. Vaccines 2018, 6, 69.

(2) Wang, J. Q.; Sun, X. Y.; Zhao, J. Y.; Yang, Y.; Cai, X. T.; Xu, J. G.; Cao, P. Exosomes: a novel strategy for treatment and prevention of diseases. Front. Pharmacol. 2017, 8, 300.

(3) Mathivanan, S.; Fahner, C. J.; Reid, G. E.; Simpson, R. J. ExoCarta 2012: database of exosomal proteins, RNA and lipids. Nucleic Acids Res. 2012, 40, D1241-D1244.

(4) Mathivanan, S.; Simpson, R. J. ExoCarta: A compendium of exosomal proteins and RNA. Proteomics 2009, 9, 4997-5000.

(5) Oksvold, M. P.; Kullmann, A.; Forfang, L.; Kierulf, B.; Li, M.; Brech, A.; Vlassov, A. V.; Smeland, E. B.; Neurauter, A.; Pedersen, K. W. Expression of B-cell surface antigens in subpopulations of exosomes released from B-cell lymphoma cells. Clin. Ther. 2014, 36, $847-862$.

(6) Théry, C. CANCER Diagnosis by extracellular vesicles. Nature 2015, 523, 161-162.

(7) Soria, F. N.; Pampliega, O.; Bourdenx, M.; Meissner, W. G.; Bezard, E.; Dehay, B. Exosomes, an unmasked culprit in neurodegenerative diseases. Front. Neurosci. 2017, 11, 26.

(8) Hartjes, T. A.; Mytnyk, S.; Jenster, G. W.; van Steijn, V.; van Royen, M. E. Extracellular vesicle quantification and characterization: common methods and emerging approaches. Bioengineering 2019, 6, 7.

(9) Rupert, D. L. M.; Claudio, V.; Lasser, C.; Bally, M. Methods for the physical characterization and quantification of extracellular vesicles in biological samples. Biochim. Biophys. Acta 2017, 1861, 3164-3179.

(10) Zabeo, D.; Cvjetkovic, A.; Lasser, C.; Schorb, M.; Lotvall, J.; Hoog, J. L. Exosomes purified from a single cell type have diverse morphology. J. Extracell. Vesicles 2017, 6, No. 1329476.

(11) Parisse, P.; Rago, I.; Severino, L. U.; Perissinotto, F.; Ambrosetti, E.; Paoletti, P.; Ricci, M.; Beltrami, A. P.; Cesselli, D.; Casalis, L. Atomic force microscopy analysis of extracellular vesicles. Eur. Biophys. J. 2017, 46, 813-820.

(12) Maas, S. L. N.; Broekman, M. L. D.; de Vrij, J. Tunable resistive pulse sensing for the characterization of extracellular vesicles. Methods Mol. Biol. 2017, 1545, 21-33.

(13) van der Vlist, E. J.; Nolte-'t Hoen, E. N. M.; Stoorvogel, W.; Arkesteijn, G. J. A.; Wauben, M. H. M. Fluorescent labeling of nanosized vesicles released by cells and subsequent quantitative and 
qualitative analysis by high-resolution flow cytometry. Nat. Protoc. 2012, 7, 1311-1326.

(14) Gercel-Taylor, C.; Atay, S.; Tullis, R. H.; Kesimer, M.; Taylor, D. D. Nanoparticle analysis of circulating cell-derived vesicles in ovarian cancer patients. Anal. Biochem. 2012, 428, 44-53.

(15) Soo, C. Y.; Song, Y. Q.; Zheng, Y.; Campbell, E. C.; Riches, A. C.; Gunn-Moore, F.; Powis, S. J. Nanoparticle tracking analysis monitors microvesicle and exosome secretion from immune cells. Immunology 2012, 136, 192-197.

(16) Fröhlich, E. The role of surface charge in cellular uptake and cytotoxicity of medical nanoparticles. Int. J. Nanomed. 2012, 7, 55775591.

(17) Bhattacharjee, S.; Rietjens, I. M. C. M.; Singh, M. P.; Atkins, T. M.; Purkait, T. K.; Xu, Z. J.; Regli, S.; Shukaliak, A.; Clark, R. J.; Mitchell, B. S.; Alink, G. M.; Marcelis, A. T. M.; Fink, M. J.; Veinot, J. G. C.; Kauzlarich, S. M.; Zuilhof, H. Cytotoxicity of surfacefunctionalized silicon and germanium nanoparticles: the dominant role of surface charges. Nanoscale 2013, 5, 4870-4883.

(18) Kaszuba, M.; Corbett, J.; Watson, F. M.; Jones, A. Highconcentration zeta potential measurements using light-scattering techniques. Philos. Trans. R. Soc., A 2010, 368, 4439-4451.

(19) Delgado, A. V.; González-Caballero, F.; Hunter, R. J.; Koopal, L. K.; Lyklema, J. Measurement and interpretation of electrokinetic phenomena. J. Colloid Interface Sci. 2007, 309, 194-224.

(20) Akagi, T.; Ichiki, T. Cell electrophoresis on a chip: what can we know from the changes in electrophoretic mobility? Anal. Bioanal. Chem. 2008, 391, 2433-2441.

(21) Bhattacharjee, S. DLS and zeta potential - what they are and what they are not? J. Controlled Release 2016, 235, 337-351.

(22) Nel, A. E.; Mädler, L.; Velegol, D.; Xia, T.; Hoek, E. M. V.; Somasundaran, P.; Klaessig, F.; Castranova, V.; Thompson, M. Understanding biophysicochemical interactions at the nano-bio interface. Nat. Mater. 2009, 8, 543-557.

(23) Li, X.; Corbett, A. L.; Taatizadeh, E.; Tasnim, N.; Little, J. P.; Garnis, C.; Daugaard, M.; Guns, E.; Hoorfar, M.; Li, I. T. S. Challenges and opportunities in exosome research-Perspectives from biology, engineering, and cancer therapy. APL Bioeng. 2019, 3, No. 011503.

(24) Osteikoetxea, X.; Sodar, B.; Nemeth, A.; Szabo-Taylor, K.; Paloczi, K.; Vukman, K. V.; Tamasi, V.; Balogh, A.; Kittel, A.; Pallinger, E.; Buzas, E. I. Differential detergent sensitivity of extracellular vesicle subpopulations. Org. Biomol. Chem. 2015, 13, 9775-9782.

(25) Filipe, V.; Hawe, A.; Jiskoot, W. Critical evaluation of nanoparticle tracking analysis (NTA) by nanosight for the measurement of nanoparticles and protein aggregates. Pharm. Res. 2010, 27, $796-810$.

(26) Kesimer, M.; Gupta, R. Physical characterization and profiling of airway epithelial derived exosomes using light scattering. Methods 2015, 87, 59-63.

(27) Sokolova, V.; Ludwig, A. K.; Hornung, S.; Rotan, O.; Horn, P. A.; Epple, M.; Glebel, B. Characterisation of exosomes derived from human cells by nanoparticle tracking analysis and scanning electron microscopy. Colloids Surf., B 2011, 87, 146-150.

(28) Baran, J.; Baj-Krzyworzeka, M.; Weglarczyk, K.; Szatanek, R.; Zembala, M.; Barbasz, J.; Czupryna, A.; Szczepanik, A.; Zembala, M. Circulating tumour-derived microvesicles in plasma of gastric cancer patients. Cancer Immunol. Immunother. 2010, 59, 841-850.

(29) Jamaludin, N. A.; Thurston, L. M.; Witek, K. J.; Meikle, A.; Basatvat, S.; Elliott, S.; Hunt, S.; Andronowska, A.; Fazeli, A. Efficient isolation, biophysical characterisation and molecular composition of extracellular vesicles secreted by primary and immortalised cells of reproductive origin. Theriogenology 2019, 135, 121-137.

(30) Bachurski, D.; Schuldner, M.; Nguyen, P.-H.; Malz, A.; Reiners, K. S.; Grenzi, P. C.; Babatz, F.; Schauss, A. C.; Hansen, H. P.; Hallek, M.; Pogge von Strandmann, E. Extracellular vesicle measurements with nanoparticle tracking analysis - an accuracy and repeatability comparison between NanoSight NS300 and ZetaView. J. Extracell. Vesicles 2019, 8, No. 1596016.
(31) Beit-Yannai, E.; Tabak, S.; Stamer, W. D. Physical exosome:exosome interactions. J. Cell. Mol. Med. 2018, 22, 20012006.

(32) György, B.; Modos, K.; Pallinger, E.; Paloczi, K.; Pasztoi, M.; Misjak, P.; Deli, M. A.; Sipos, A.; Szalai, A.; Voszka, I.; Polgar, A.; Toth, K.; Csete, M.; Nagy, G.; Gay, S.; Falus, A.; Kittel, A.; Buzas, E. I. Detection and isolation of cell-derived microparticles are compromised by protein complexes resulting from shared biophysical parameters. Blood 2011, 117, E39-E48.

(33) Hsu, J.-P.; Liu, B.-T. Effect of particle size on critical coagulation concentration. J. Colloid Interface Sci. 1998, 198, 186189.

(34) Zhang, Z.; Zhao, L.; Li, Y.; Chu, M. A modified method to calculate critical coagulation concentration based on DLVO theory. Math. Probl. Eng. 2015, 2015, No. 317483.

(35) Galli, M.; Saringer, S.; Szilagyi, I.; Trefalt, G. A simple method to determine critical coagulation concentration from electrophoretic mobility, arXiv:2003.12513. arXiv.org e-Print archive. https://ui. adsabs.harvard.edu/abs/2020arXiv200312513G (accessed May 01, 2020).

(36) Moore, T. L.; Rodriguez-Lorenzo, L.; Hirsch, V.; Balog, S.; Urban, D.; Jud, C.; Rothen-Rutishauser, B.; Lattuada, M.; Petri-Fink, A. Nanoparticle colloidal stability in cell culture media and impact on cellular interactions. Chem. Soc. Rev. 2015, 44, 6287-6305.

(37) Tadros, T. General Principles of Colloid Stability and the Role of Surface Forces. In Colloid Stability; Tadros, T. F., Eds.; Wiley-VCH, 2011; pp 1-22.

(38) Okur, H. I.; Hladílková, J.; Rembert, K. B.; Cho, Y.; Heyda, J.; Dzubiella, J.; Cremer, P. S.; Jungwirth, P. Beyond the Hofmeister Series: ion-specific effects on proteins and their biological functions. J. Phys. Chem. B 2017, 121, 1997-2014.

(39) Muráth, S.; Sáringer, S.; Somosi, Z.; Szilágyi, I. Effect of ionic compounds of different valences on the stability of titanium oxide colloids. Colloids Interfaces 2018, 2, 32.

(40) Salgin, S.; Salgin, U.; Bahadir, S. Zeta potentials and isoelectric points of biomolecules: the effects of ion types and ionic strengths. Int. J. Electrochem. Sci. 2012, 7, 12404-12414.

(41) Cheng, Y. R.; Zeng, Q. Y.; Han, Q.; Xia, W. L. Effect of pH, temperature and freezing-thawing on quantity changes and cellular uptake of exosomes. Protein Cell 2019, 10, 295-299.

(42) Zhao, Y.; Chen, K. Y.; Li, H. N.; Wu, H. Y. Effect of pH on the isolation of urinary exosome. Int. Urol. Nephrol. 2017, 49, 165-169.

(43) Van Deun, J.; Mestdagh, P.; Agostinis, P.; Akay, Ö.; Anand, S.; Anckaert, J.; Martinez, Z. A.; Baetens, T.; Beghein, E.; Bertier, L.; Berx, G.; Boere, J.; Boukouris, S.; Bremer, M.; Buschmann, D.; Byrd, J. B.; Casert, C.; Cheng, L.; Cmoch, A.; Daveloose, D.; De Smedt, E.; Demirsoy, S.; Depoorter, V.; Dhondt, B.; Driedonks, T. A. P.; Dudek, A.; Elsharawy, A.; Floris, I.; Foers, A. D.; Gärtner, K.; Garg, A. D.; Geeurickx, E.; Gettemans, J.; Ghazavi, F.; Giebel, B.; Kormelink, T. G.; Hancock, G.; Helsmoortel, H.; Hill, A. F.; Hyenne, V.; Kalra, H.; Kim, D.; Kowal, J.; Kraemer, S.; Leidinger, P.; Leonelli, C.; Liang, Y.; Lippens, L.; Liu, S.; Lo Cicero, A.; Martin, S.; Mathivanan, S.; Mathiyalagan, P.; Matusek, T.; Milani, G.; Monguió-Tortajada, M.; Mus, L. M.; Muth, D. C.; Németh, A.; Nolte-'t Hoen, E. N. M.; O’Driscoll, L.; Palmulli, R.; Pfaffl, M. W.; Primdal-Bengtson, B.; Romano, E.; Rousseau, Q.; Sahoo, S.; Sampaio, N.; Samuel, M.; Scicluna, B.; Soen, B.; Steels, A.; Swinnen, J. V.; Takatalo, M.; Thaminy, S.; Théry, C.; Tulkens, J.; Van Audenhove, I.; van der Grein, S.; Van Goethem, A.; van Herwijnen, M. J.; Van Niel, G.; Van Roy, N.; Van Vliet, A. R.; Vandamme, N.; Vanhauwaert, S.; Vergauwen, G.; Verweij, F.; Wallaert, A.; Wauben, M.; Witwer, K. W.; Zonneveld, M. I.; De Wever, O.; Vandesompele, J.; Hendrix, A.; Consortium, E.-T. EV-TRACK: transparent reporting and centralizing knowledge in extracellular vesicle research. Nat. Methods 2017, 14, $228-232$.

(44) Kornilov, R.; Puhka, M.; Mannerstrom, B.; Hiidenmaa, H.; Peltoniemi, H.; Siljander, P.; Seppanen-Kaijansinkko, R.; Kaur, S. Efficient ultrafiltration-based protocol to deplete extracellular vesicles from fetal bovine serum. J. Extracell. Vesicles 2018, 7, No. 1422674. 
(45) Théry, C.; Witwer, K. W.; Aikawa, E.; Alcaraz, M. J.; Anderson, J. D.; Andriantsitohaina, R.; Antoniou, A.; Arab, T.; Archer, F.; AtkinSmith, G. K.; Ayre, D. C.; Bach, J.-M.; Bachurski, D.; Baharvand, H.; Balaj, L.; Baldacchino, S.; Bauer, N. N.; Baxter, A. A.; Bebawy, M.; Beckham, C.; Bedina Zavec, A.; Benmoussa, A.; Berardi, A. C.; Bergese, P.; Bielska, E.; Blenkiron, C.; Bobis-Wozowicz, S.; Boilard, E.; Boireau, W.; Bongiovanni, A.; Borràs, F. E.; Bosch, S.; Boulanger, C. M.; Breakefield, X.; Breglio, A. M.; Brennan, M. Á.; Brigstock, D. R.; Brisson, A.; Broekman, M. L. D.; Bromberg, J. F.; Bryl-Górecka, P.; Buch, S.; Buck, A. H.; Burger, D.; Busatto, S.; Buschmann, D.; Bussolati, B.; Buzás, E. I.; Byrd, J. B.; Camussi, G.; Carter, D. R. F.; Caruso, S.; Chamley, L. W.; Chang, Y.-T.; Chen, C.; Chen, S.; Cheng, L.; Chin, A. R.; Clayton, A.; Clerici, S. P.; Cocks, A.; Cocucci, E.; Coffey, R. J.; Cordeiro-da-Silva, A.; Couch, Y.; Coumans, F. A. W.; Coyle, B.; Crescitelli, R.; Criado, M. F.; D'Souza-Schorey, C.; Das, S.; Datta Chaudhuri, A.; de Candia, P.; De Santana, E. F.; De Wever, O.; del Portillo, H. A.; Demaret, T.; Deville, S.; Devitt, A.; Dhondt, B.; Di Vizio, D.; Dieterich, L. C.; Dolo, V.; Dominguez Rubio, A. P.; Dominici, M.; Dourado, M. R.; Driedonks, T. A. P.; Duarte, F. V.; Duncan, H. M.; Eichenberger, R. M.; Ekström, K.; El Andaloussi, S.; Elie-Caille, C.; Erdbrügger, U.; Falcón-Pérez, J. M.; Fatima, F.; Fish, J. E.; Flores-Bellver, M.; Försönits, A.; Frelet-Barrand, A.; Fricke, F.; Fuhrmann, G.; Gabrielsson, S.; Gámez-Valero, A.; Gardiner, C.; Gärtner, K.; Gaudin, R.; Gho, Y. S.; Giebel, B.; Gilbert, C.; Gimona, M.; Giusti, I.; Goberdhan, D. C. I.; Görgens, A.; Gorski, S. M.; Greening, D. W.; Gross, J. C.; Gualerzi, A.; Gupta, G. N.; Gustafson, D.; Handberg, A.; Haraszti, R. A.; Harrison, P.; Hegyesi, H.; Hendrix, A.; Hill, A. F.; Hochberg, F. H.; Hoffmann, K. F.; Holder, B.; Holthofer, H.; Hosseinkhani, B.; Hu, G.; Huang, Y.; Huber, V.; Hunt, S.; Ibrahim, A. G.-E.; Ikezu, T.; Inal, J. M.; Isin, M.; Ivanova, A.; Jackson, H. K.; Jacobsen, S.; Jay, S. M.; Jayachandran, M.; Jenster, G.; Jiang, L.; Johnson, S. M.; Jones, J. C.; Jong, A.; Jovanovic-Talisman, T.; Jung, S.; Kalluri, R.; Kano, S.-i.; Kaur, S.; Kawamura, Y.; Keller, E. T.; Khamari, D.; Khomyakova, E.; Khvorova, A.; Kierulf, P.; Kim, K. P.; Kislinger, T.; Klingeborn, M.; Klinke, D. J.; Kornek, M.; Kosanović, M. M.; Kovács, ÁF.; Krämer-Albers, E.-M.; Krasemann, S.; Krause, M.; Kurochkin, I. V.; Kusuma, G. D.; Kuypers, S.; Laitinen, S.; Langevin, S. M.; Languino, L. R.; Lannigan, J.; Lässer, C.; Laurent, L. C.; Lavieu, G.; Lázaro-Ibáñez, E.; Le Lay, S.; Lee, M.-S.; Lee, Y. X. F.; Lemos, D. S.; Lenassi, M.; Leszczynska, A.; Li, I. T. S.; Liao, K.; Libregts, S. F.; Ligeti, E.; Lim, R.; Lim, S. K.; Linē, A.; Linnemannstöns, K.; Llorente, A.; Lombard, C. A.; Lorenowicz, M. J.; Lörincz, ÁM.; Lötvall, J.; Lovett, J.; Lowry, M. C.; Loyer, X.; Lu, Q.; Lukomska, B.; Lunavat, T. R.; Maas, S. L. N.; Malhi, H.; Marcilla, A.; Mariani, J.; Mariscal, J.; Martens-Uzunova, E. S.; Martin-Jaular, L.; Martinez, M. C.; Martins, V. R.; Mathieu, M.; Mathivanan, S.; Maugeri, M.; McGinnis, L. K.; McVey, M. J.; Meckes, D. G.; Meehan, K. L.; Mertens, I.; Minciacchi, V. R.; Möller, A.; Møller Jørgensen, M.; Morales-Kastresana, A.; Morhayim, J.; Mullier, F.; Muraca, M.; Musante, L.; Mussack, V.; Muth, D. C.; Myburgh, K. H.; Najrana, T.; Nawaz, M.; Nazarenko, I.; Nejsum, P.; Neri, C.; Neri, T.; Nieuwland, R.; Nimrichter, L.; Nolan, J. P.; Nolte-'t Hoen, E. N. M.; Noren Hooten, N.; O'Driscoll, L.; O'Grady, T.; O’Loghlen, A.; Ochiya, T.; Olivier, M.; Ortiz, A.; Ortiz, L. A.; Osteikoetxea, X.; Østergaard, O.; Ostrowski, M.; Park, J.; Pegtel, D. M.; Peinado, H.; Perut, F.; Pfaffl, M. W.; Phinney, D. G.; Pieters, B. C. H.; Pink, R. C.; Pisetsky, D. S.; Pogge von Strandmann, E.; Polakovicova, I.; Poon, I. K. H.; Powell, B. H.; Prada, I.; Pulliam, L.; Quesenberry, P.; Radeghieri, A.; Raffai, R. L.; Raimondo, S.; Rak, J.; Ramirez, M. I.; Raposo, G.; Rayyan, M. S.; Regev-Rudzki, N.; Ricklefs, F. L.; Robbins, P. D.; Roberts, D. D.; Rodrigues, S. C.; Rohde, E.; Rome, S.; Rouschop, K. M. A.; Rughetti, A.; Russell, A. E.; Saá, P.; Sahoo, S.; Salas-Huenuleo, E.; Sánchez, C.; Saugstad, J. A.; Saul, M. J.; Schiffelers, R. M.; Schneider, R.; Schøyen, T. H.; Scott, A.; Shahaj, E.; Sharma, S.; Shatnyeva, O.; Shekari, F.; Shelke, G. V.; Shetty, A. K.; Shiba, K.; Siljander, P. R. M.; Silva, A. M.; Skowronek, A.; Snyder, O. L.; Soares, R. P.; Sódar, B. W.; Soekmadji, C.; Sotillo, J.; Stahl, P. D.; Stoorvogel, W.; Stott, S. L.; Strasser, E. F.; Swift, S.; Tahara, H.; Tewari, M.; Timms, K.; Tiwari, S.; Tixeira, R.; Tkach, M.; Toh, W. S.; Tomasini, R.; Torrecilhas, A. C.; Tosar, J. P.;
Toxavidis, V.; Urbanelli, L.; Vader, P.; van Balkom, B. W. M.; van der Grein, S. G.; Van Deun, J.; van Herwijnen, M. J. C.; Van KeurenJensen, K.; van Niel, G.; van Royen, M. E.; van Wijnen, A. J.; Vasconcelos, M. H.; Vechetti, I. J.; Veit, T. D.; Vella, L. J.; Velot, E.; Verweij, F. J.; Vestad, B.; Viñas, J. L.; Visnovitz, T.; Vukman, K. V.; Wahlgren, J.; Watson, D. C.; Wauben, M. H. M.; Weaver, A.; Webber, J. P.; Weber, V.; Wehman, A. M.; Weiss, D. J.; Welsh, J. A.; Wendt, S.; Wheelock, A. M.; Wiener, Z.; Witte, L.; Wolfram, J.; Xagorari, A.; Xander, P.; Xu, J.; Yan, X.; Yáñez-Mó, M.; Yin, H.; Yuana, Y.; Zappulli, V.; Zarubova, J.; Žèkas, V.; Zhang, J.-y.; Zhao, Z.; Zheng, L.; Zheutlin, A. R.; Zickler, A. M.; Zimmermann, P.; Zivkovic, A. M.; Zocco, D.; Zuba-Surma, E. K. Minimal information for studies of extracellular vesicles 2018 (MISEV2018): a position statement of the International Society for extracellular vesicles and update of the MISEV2014 guidelines. J. Extracell. Vesicles 2018, 7, No. 1535750.

(46) Es-Haghi, M.; Godakumara, K.; Häling, A.; Lättekivi, F.; Lavrits, A.; Viil, J.; Andronowska, A.; Nafee, T.; James, V.; Jaakma, Ü.; Salumets, A.; Fazeli, A. Specific trophoblast transcripts transferred by extracellular vesicles affect gene expression in endometrial epithelial cells and may have a role in embryo-maternal crosstalk. Cell Commun. Signaling 2019, 17, 146. 